\title{
Theoretical therapeutic impact of lymph node dissection on adenocarcinoma and squamous cell carcinoma of the esophagogastric junction
}

\author{
Takaki Yoshikawa $\cdot$ Hiroya Takeuchi $\cdot$ Shinichi Hasegawa $\cdot$ Isao Nozaki $\cdot$ \\ Kentaro Kishi · Seiji Ito · Masaki Ohi · Shinji Mine · Johji Hara • \\ Tatsuo Matsuda • Naoki Hiki • Yukinori Kurokawa
}

Received: 12 May 2014/ Accepted: 28 October 2014/Published online: 21 November 2014

(C) The International Gastric Cancer Association and The Japanese Gastric Cancer Association 2014

\begin{abstract}
Backgrounds The aim of this study was to evaluate the theoretical therapeutic impact of dissecting each lymph node station for adenocarcinoma and squamous cell carcinoma of the esophagogastric junction.

Methods This multicenter study included 431 junctional cancer patients (381 adenocarcinomas and 50 squamous cell carcinomas) who fulfilled the following criteria: (1) the center of the tumor was located between $1 \mathrm{~cm}$ above and $2 \mathrm{~cm}$ below the esophagogastric junction, and (2) the tumor invaded the junction. The theoretical therapeutic impact of dissecting each lymph node station was evaluated based on the therapeutic value index calculated by multiplying the frequency of metastasis to each station and the 5-year survival rate of patients with metastasis to that station.
\end{abstract}

T. Yoshikawa, H. Takeuchi and S. Hasegawa contributed equally to this article.

T. Yoshikawa $\cdot$ S. Hasegawa

Department of Gastrointestinal Surgery, Kanagawa Cancer

Center, Yokohama, Japan

H. Takeuchi - T. Matsuda

Department of Surgery, Keio University School of Medicine,

Tokyo, Japan

I. Nozaki

Department of Surgery, Shikoku Cancer Center, Matsuyama, Japan

K. Kishi

Department of Surgery, Osaka Medical Center for Cancer and Cardiovascular Diseases, Osaka, Japan

S. Ito

Department of Gastroenterological Surgery, Aichi Cancer

Center, Nagoya, Japan
Results The 5-year overall survival rates (95\% confidence interval) were $60.4 \%(55.1-65.7)$ in the adenocarcinoma cases and $52.3 \%(35.6-69.0)$ in the squamous cell carcinoma cases. The nodal stations showing the first to fifth highest index were the paracardial and lesser curvature nodes (nos. 1, 2 and 3), nodes at the root of the left gastric artery (no. 7) and lower mediastinal lymph nodes, regardless of the histology.

Conclusions Nodal dissection achieved by proximal gastrectomy and lower esophagectomy should be the minimal requirement for junctional cancer regardless of the histology, considering the therapeutic value indices for the relevant lymph node stations.

Keywords Gastric carcinoma - Esophageal carcinoma . Lymph node metastasis - Surgical therapy

M. Ohi

Department of Innovative Surgery, Mie University Graduate School of Medicine, Tsu, Mie, Japan

S. Mine $\cdot$ N. Hiki $(\square)$

Department of Surgery, Cancer Institute Ariake Hospital,

3-8-31 Ariake, Koto-ku, Tokyo 135-8550, Japan

e-mail: naoki.hiki@jfcr.or.jp

J. Hara · Y. Kurokawa ( $₫)$

Department of Gastroenterological Surgery, Osaka University

Graduate School of Medicine, 2-2, E2, Yamadaoka, Suita,

Osaka 565-0871, Japan

e-mail: ykurokawa@gesurg.med.osaka-u.ac.jp 


\section{Introduction}

Carcinomas of the esophagus and esophagogastric junction have become a major topic in both Western and Eastern countries. In Western countries, the incidence of the adenocarcinoma (AC) has increased rapidly, whereas that of squamous cell carcinoma has gradually decreased [1-5]. On the other hand, squamous cell carcinoma (SCC) is still the major histological type in Asian countries $[6,7]$.

$\mathrm{AC}$ arises from the esophageal gland, specialized intestinal epithelial metaplasia (so-called Barrett's esophagus) or the gastric mucosa, such as the fundic gland, while SCC develops from the squamous cells of the esophageal mucosa. The most common location of $\mathrm{AC}$ is the lower esophagus to the junction in Western countries and the subcardia in Eastern countries. On the other hand, SCC mostly occurs at the thoracic esophagus, but sometimes arises from the abdominal esophagus close to the junction.

Reflecting the differences in the dominant location, most patients with $\mathrm{AC}$ undergo resection of the lower esophagus and stomach, while those with SCC receive subtotal esophagectomy. The extent of lymph node dissection is determined by the type of surgery and the surgical field. The different surgical strategies used for AC and the SCC affect the selection of the surgery for junctional cancer. However, it remains unclear whether the appropriate nodal dissection differs depending on the histology.

To explore the optimal surgical strategy for junctional cancer, we conducted this multiinstitutional study to compare the theoretical therapeutic impact of dissecting each lymph node station between junctional AC and SCC.

\section{Patients and methods}

Patients

This study included 431 junctional cancer patients, consisting of 381 cases of adenocarcinoma (AC) and 50 cases of squamous cell carcinoma (SCC). All patients underwent $\mathrm{R} 0$ resection at one of seven institutions in Japan between March 1986 and October 2010. All tumors fulfilled the following criteria: (1) histologically proven adenocarcinoma or squamous cell carcinoma, (2) the center of the tumor was located between $1 \mathrm{~cm}$ above and $2 \mathrm{~cm}$ below the esophagogastric junction and (3) tumors invaded the junction. AC that fulfilled the above criteria corresponded to Siewert type II tumors [8, 9]. The type of surgical approach, esophagectomy vs. gastrectomy, was not specified in this study. All patients underwent R0 resection at one of seven institutions in Japan between March 1986 and October 2010. Extent of nodal dissection and indication to dissect M1 disease were determined by the policy of each institution and were not standardized among the study group. This study (HIK-01) was approved by the Steering Committee of the Young Gastric Surgeons Research Group in Japan.

Evaluation and statistical considerations

The progression of the tumors was evaluated by the UICCTNM, 7th edition [10]. The station-based classification of the abdominal field adhered to the criteria defined by the Japanese Gastric Cancer Association [11] (Table 1). Mediastinal nodes were classified into three regions: upper, middle and lower. The definition of the extent of the abdominal lymph node dissection (D1+, D2 and D2+) was based on the Japanese Gastric Cancer Treatment Guidelines, version 3 [12]. To evaluate the theoretical therapeutic impact of dissecting each lymph node station, we adopted a method using the therapeutic value index introduced by Sasako et al. [13] in 1995. The index was calculated by multiplying the incidence of metastasis to the station by the 5-year survival rate of patients (defined as a percentage). Although the current treatment guidelines do not recommend dissection of M1 disease, inclusion of at least a part

Table 1 Lymph node stations

\begin{tabular}{|c|c|}
\hline No. 1 & Right paracardial nodes \\
\hline No. 2 & Left paracardial nodes \\
\hline No. 3 & Lesser curvature nodes \\
\hline No. 4sa & Left greater curvature nodes along the short gastric arteries \\
\hline No. $4 \mathrm{sb}$ & $\begin{array}{l}\text { Left greater curvature nodes along the left gastroepiploic } \\
\text { artery }\end{array}$ \\
\hline No. $4 \mathrm{~d}$ & $\begin{array}{l}\text { Right greater curvature nodes along the right } \\
\text { gastroepiploic artery }\end{array}$ \\
\hline No. 5 & Suprapyloric nodes \\
\hline No. 6 & Infrapyloric nodes \\
\hline No. 7 & Nodes at the root of the left gastric artery \\
\hline No. $8 \mathrm{a}$ & Nodes along the common hepatic artery \\
\hline No. 9 & Nodes at the celiac artery \\
\hline No. 10 & Nodes around the splenic hilum \\
\hline No. $11 \mathrm{p}$ & Nodes along the proximal splenic artery \\
\hline No. $11 \mathrm{~d}$ & Nodes along the distal splenic artery \\
\hline No. $12 \mathrm{a}$ & Nodes along the proper hepatic artery \\
\hline No. $16 \mathrm{a} 2$ & $\begin{array}{l}\text { Paraaortic nodes located between the upper margin of the } \\
\text { origin of the celiac artery and the lower border of the } \\
\text { left renal vein }\end{array}$ \\
\hline No. $16 b 1$ & $\begin{array}{l}\text { Paraaortic nodes located between the lower border of the } \\
\text { left renal vein and the upper border of the origin of the } \\
\text { inferior mesenteric artery }\end{array}$ \\
\hline No. 19 & Infradiaphragmatic nodes \\
\hline No. 20 & $\begin{array}{l}\text { Paraesophageal nodes at the esophageal hiatus of the } \\
\text { diaphragm }\end{array}$ \\
\hline
\end{tabular}


of the paraaortic lymph nodes (no. 16a2) as a standard of care for the treatment of junctional adenocarcinoma remains a matter of controversy in Japan. Metastasis to the paraaortic node is currently defined as M1 disease, and, in order to evaluate the index for paraaortic lymph nodes, inclusion of patients with M1 disease who underwent R0 resection was mandatory in this study.

All data are presented as the medians and total ranges, unless otherwise stated. The chi-square test was used to compare the proportions of the patients. The survival rates were calculated by the Kaplan-Meier method and compared by a log-rank test.

\section{Results}

Patients

A total of 431 patients, consisting of 381 with $\mathrm{AC}$ and 50 with squamous cell carcinoma (SCC), fulfilled the eligibility criteria and were enrolled in the study. Table 2 describes a comparison of the background characteristics of the AC and SCC patients. The distance between the tumor center and EGJ was significantly different between the AC and the SCC groups, but the difference was less than $10 \mathrm{~mm}$. pT1 was noted in $20.7 \%(79 / 381)$ of patients in the AC group, while it was noted in only $4.0 \%(2 / 50)$ of patients in the SCC group. Lymph node metastasis was observed in $64.3 \%(245 / 381)$ of patients in the AC group and $76.0 \%(38 / 50)$ in the SCC group. M1 disease was observed in $6.8 \%(26 / 381)$ of the AC patients, including nonregional lymph nodes in 23 patients, liver metastasis in 3 and peritoneal dissemination in 3, while it was observed in $4 \%(2 / 50)$ of the patients in the SCC group, including lung metastasis in 1 and unknown in 1.

Total gastrectomy and lower/abdominal esophagectomy via a transhiatal approach were the most common procedures used in patients with AC, while total/subtotal esophagectomy via a right thoracic approach and proximal gastrectomy were most commonly performed in patients with SCC. Splenectomy was frequently performed in the AC group, while neoadjuvant chemotherapy was frequently administered in the SCC group.

The median follow-up period of the survivors exceeded 5 years (60.4 months). The 5-year overall survival rates (95\% confidence interval) were $60.4 \%(55.1-65.7)$ in the AC group and 52.3\% (35.6-69.0) in the SCC group.

Calculated index for each nodal station (Table 3)

Table 3 describes the incidence of nodal metastasis, 5-year survival rate and calculated therapeutic index for each histology. As reliability of the index was low in the nodes that were not frequently dissected, the index was only calculated for nodes that were dissected in 20 or more patients. For this reason, we excluded the upper mediastinal nodes and cervical nodes for the AC group and the nodes along the greater curvature (nos. 4sa, 4sb, 4d, and 6), nodes of the right gastric artery (no. 5), nodes along the splenic artery and around the spleen (nos. 10 and 11d), nodes along the proper hepatic artery (no. 12a), paraaortic nodes (no. 16) and cervical nodes for the SC group.

In the $\mathrm{AC}$ group, the nodal station showing the highest index was the lesser curvature nodes (nos. 3, 19.8), followed by the right paracardial nodes (nos. 1, 16.2), left paracardial nodes (nos. 2, 13.6), nodes at the root of the left gastric artery (nos. 7, 11.7) and the lower mediastinal lymph nodes (6.0). In the SCC group, the nodal station showing the highest index was the right paracardial nodes (nos. 1, 17.1), followed by the left paracardial nodes (nos. 2, 7.4), lesser curvature nodes (nos. 3, 7.2), nodes at the root of the left gastric artery (nos. 7, 4.4) and lower mediastinal lymph nodes (4.2). Thus, nos. 1, 2, 3, 7 and the lower mediastinal lymph nodes were the stations with the highest priority for dissection, regardless of histological type.

The subsequent nodes in terms of the calculated index were the middle mediastinal nodes (5.0), nodes along the proximal splenic artery (nos. $11 \mathrm{p}, 4.7$ ), paraaortic nodes located between the upper margin of the origin of the celiac artery and lower border of the left renal vein (nos. 16a2, 2.4), nodes around the splenic hilum (nos. 10, 1.8), nodes at the celiac artery (nos. 9, 1.7) and nodes along the distal splenic artery (nos. 11d, 1.7) in the AC group; however, the index for all of these nodes was 0 in the SCC group, except for the no. 9 node (2.4) and nodes 10,11d and 16, which were not calculated.

\section{Discussion}

The present study demonstrated that the lymph node stations with high theoretical therapeutic impact for junctional cancer were the same regardless of the histology. This result suggested that it is not necessary to change the surgical strategy based on the histological type. Proximal gastrectomy and lower esophagectomy should be the minimal requirement to dissect the lymph nodes with the first to fifth highest indices, regardless of the histology of the primary tumor.

In this study, we adopted the therapeutic value index to evaluate the theoretical therapeutic impact of dissecting each lymph node station. This index includes the prognosis of the patients with metastasis to the station, as well as the metastatic incidence. Using this method, we compared the the theoretical therapeutic impact of dissecting each lymph 
Table 2 Background characteristics of the AC and SCC patients

\begin{tabular}{|c|c|c|c|c|c|}
\hline \multirow{3}{*}{ Age (years) } & \multicolumn{2}{|l|}{$\mathrm{AC}(n=381)$} & \multicolumn{2}{|l|}{$\operatorname{SCC}(n=50)$} & \multirow[t]{2}{*}{$P$ value } \\
\hline & Number of patients ${ }^{\mathrm{a}}$ & $\%$ & Number of patients ${ }^{\mathrm{a}}$ & $\%$ & \\
\hline & & & & & 0.402 \\
\hline Median (range) & $63(18-88)$ & - & $66(42-78)$ & - & \\
\hline Gender & & & & & 0.734 \\
\hline Male & 305 & 80.1 & 39 & 78.0 & \\
\hline Female & 76 & 19.9 & 11 & 22.0 & \\
\hline Tumor size $(\mathrm{mm})$ & & & & & 0.348 \\
\hline Median (range) & $50(10-180)$ & - & $47(5-125)$ & - & \\
\hline Center of the tumor from EGJ $(\mathrm{mm})$ & & & & & $<0.001$ \\
\hline Median (range) & $+7.5(-10$ to +20$)$ & - & $0(-10$ to +17.5$)$ & - & \\
\hline Histopathological type & & & & & 0.717 \\
\hline Differentiated & 259 & 68.0 & 35 & 70.0 & \\
\hline Undifferentiated & 117 & 30.7 & 14 & 28.0 & \\
\hline Unknown & 5 & 1.3 & 1 & 2.0 & \\
\hline pT category & & & & & $<0.001$ \\
\hline pT1 & 79 & 20.7 & 2 & 4.0 & \\
\hline pT2 & 56 & 14.7 & 7 & 14.0 & \\
\hline pT3 & 137 & 36.0 & 36 & 72.0 & \\
\hline pT4 & 109 & 28.6 & 5 & 10.0 & \\
\hline $\mathrm{pN}$ category & & & & & 0.111 \\
\hline pNO & 136 & 35.7 & 12 & 24.0 & \\
\hline $\mathrm{pN} 1$ & 79 & 20.7 & 17 & 34.0 & \\
\hline $\mathrm{pN} 2$ & 86 & 22.6 & 13 & 26.0 & \\
\hline $\mathrm{pN} 3$ & 80 & 21.0 & 8 & 16.0 & \\
\hline M category & & & & & 0.446 \\
\hline M0 & 355 & 93.2 & 48 & 96.0 & \\
\hline M1 & 26 & 6.8 & 2 & 4.0 & \\
\hline Number of metastatic lymph nodes & & & & & 0.686 \\
\hline Median (range) & $2(0-52)$ & - & $2(0-18)$ & - & \\
\hline Approach & & & & & $<0.001$ \\
\hline Right transthoracic & 34 & 8.9 & 40 & 80.0 & \\
\hline Left transthoracic & 99 & 26.0 & 5 & 10.0 & \\
\hline Transhiatal & 248 & 65.1 & 5 & 10.0 & \\
\hline Type of esophageal resection & & & & & $<0.001$ \\
\hline Total/subtotal esophagectomy & 27 & 7.1 & 38 & 76.0 & \\
\hline Lower/abdominal esophagectomy & 354 & 92.9 & 12 & 24.0 & \\
\hline Type of gastric resection & & & & & $<0.001$ \\
\hline Total gastrectomy & 264 & 69.3 & 6 & 12.0 & \\
\hline Proximal gastrectomy & 117 & 30.7 & 44 & 88.0 & \\
\hline Splenectomy & & & & & $<0.001$ \\
\hline Yes & 215 & 56.4 & 6 & 12.0 & \\
\hline No & 166 & 43.6 & 44 & 88.0 & \\
\hline Neoadjuvant chemotherapy & & & & & $<0.001$ \\
\hline Yes & 41 & 10.8 & 28 & 56.0 & \\
\hline No & 340 & 89.2 & 22 & 44.0 & \\
\hline Adjuvant chemotherapy & & & & & 0.773 \\
\hline Yes & 122 & 32.0 & 15 & 30.0 & \\
\hline No & 259 & 68.0 & 35 & 70.0 & \\
\hline
\end{tabular}

${ }^{a}$ Except age, tumor size, center of the tumor from the EGJ and number of metastatic nodes 
Table 3 The incidence of nodal metastasis, 5-year survival rate and calculated therapeutic value index

\begin{tabular}{|c|c|c|c|c|c|c|}
\hline & \multicolumn{2}{|c|}{ Metastatic incidence (\%) } & \multicolumn{2}{|c|}{$\begin{array}{l}5 \text {-year survival rate of patients with metastasis (95\% } \\
\text { confidence interval) }\end{array}$} & \multicolumn{2}{|c|}{$\begin{array}{l}\text { Therapeutic value } \\
\text { index }\end{array}$} \\
\hline & $\mathrm{AC}$ & $\mathrm{SCC}$ & $\mathrm{AC}$ & SCC & $\mathrm{AC}$ & $\mathrm{SCC}$ \\
\hline No. 1 & $147 / 369(39.8)$ & $21 / 47(44.7)$ & $40.7(32.1-49.3)$ & $38.3(16.0-60.6)$ & 16.2 & 17.1 \\
\hline No. 2 & $122 / 364(30.8)$ & $12 / 48(25.0)$ & $44.3(34.7-53.9)$ & $29.6(0-61.0)$ & 13.6 & 7.4 \\
\hline No. 3 & $156 / 376(41.5)$ & $17 / 49(34.7)$ & $47.8(39.4-56.2)$ & $20.8(0-52.2)$ & 19.8 & 7.2 \\
\hline No. 4 sa & $14 / 326(4.3)$ & $0 / 8(0)$ & $23.1(0.2-46.0)$ & NA & 1.0 & NA \\
\hline No. $4 \mathrm{sb}$ & $8 / 298(2.7)$ & $0 / 7(0)$ & $12.5(0-35.4)$ & NA & 0.3 & NA \\
\hline No. $4 \mathrm{~d}$ & $8 / 280(2.9)$ & $1 / 6(16.7)$ & $37.5(4.0-71.0)$ & NA & 1.1 & NA \\
\hline No. 5 & $4 / 239(1.7)$ & $0 / 6(0)$ & 0 & NA & 0 & NA \\
\hline No. 6 & $2 / 258(0.8)$ & $0 / 6(0)$ & $50.0(0-100)$ & NA & 0.4 & NA \\
\hline No. 7 & 98/367 (26.7) & $8 / 46(17.4)$ & $43.9(33.5-54.3)$ & $25.0(0-55.0)$ & 11.7 & 4.4 \\
\hline No. 8a & $16 / 325(4.9)$ & $1 / 42(2.4)$ & $13.6(0-31.0)$ & 0 & 0.7 & 0 \\
\hline No. 9 & $35 / 300(11.7)$ & $3 / 42(7.1)$ & $14.4(2.1-26.7)$ & $33.3(0-86.6)$ & 1.7 & 2.4 \\
\hline No. 10 & $21 / 221(9.5)$ & $0 / 6(0)$ & $19.0(2.1-35.9)$ & NA & 1.8 & NA \\
\hline No. $11 \mathrm{p}$ & $53 / 309(17.2)$ & $1 / 34(2.9)$ & $27.4(14.1-40.7)$ & 0 & 4.7 & 0 \\
\hline No. $11 \mathrm{~d}$ & $11 / 176(6.3)$ & $0 / 7(0)$ & $26.5(0-56.7)$ & NA & 1.7 & NA \\
\hline No. $12 \mathrm{a}$ & $1 / 72(1.4)$ & $1 / 4(25.0)$ & 0 & 0 & 0 & NA \\
\hline No. $16 a 2$ & $18 / 125(14.4)$ & $1 / 9(11.1)$ & $16.7(0-33.9)$ & 0 & 2.4 & NA \\
\hline No. $16 b 1$ & $6 / 32(18.8)$ & $1 / 6(16.7)$ & 0 & 0 & 0 & NA \\
\hline No. 19 & $7 / 143(4.9)$ & $1 / 16(6.3)$ & 0 & 0 & 0 & NA \\
\hline No. 20 & $2 / 137(1.5)$ & $0 / 23(0)$ & 0 & 0 & 0 & 0 \\
\hline Lower mediastinal nodes & 34/188 (18.1) & $12 / 48(25.0)$ & $33.2(16.0-50.4)$ & $16.7(0-44.9)$ & 6.0 & 4.2 \\
\hline Middle mediastinal nodes & $8 / 40(20.0)$ & $8 / 42(19.0)$ & $25.0(0-55.0)$ & 0 & 5.0 & 0 \\
\hline Upper mediastinal nodes & 3/19 (15.8) & $3 / 36(8.3)$ & 0 & 0 & NA & 0 \\
\hline Cervical nodes & $2 / 10(20.0)$ & $3 / 19(15.8)$ & 0 & 0 & NA & NA \\
\hline
\end{tabular}

$A C$ adenocarcinoma; SCC squamous cell carcinoma; NA not available (expressed as NA when 5-year survival rate could not be calculated)

node station between junctional AC and SCC. The nodal stations showing the first to fifth highest index values were the same regardless of the histological type: the paracardial and lesser curvature nodes (nos. 1, 2 and 3), nodes at the root of the left gastric artery (no. 7) and lower mediastinal lymph nodes. These results were mostly consistent with the previous studies of junctional AC [14-18] and with the recently published study by Yabusaki et al. [19] except for the lower mediastinal lymph nodes. In their study, the index for the lower mediastinal nodes was 6.9 in total for SCC, which exceeded the value of nos. 2 and 7. On the other hand, that for AC was reportedly low at 2.9. However, dissected cases for AC were less than 10 for the paraesophageal nodes in the lower mediastinum in their study, which suggested low reliability of the value for low mediastinal nodes in AC.

So far, no report has evaluated the theoretical therapeutic impact of dissecting each lymph node station of junctional squamous cell carcinoma. However, Kakeji et al. [20] previously demonstrated the details of the metastatic incidence to the abdominal cavity in junctional squamous cell carcinoma. The nodal stations that showed the first to fourth highest metastatic incidence were nos. 1, 2, 3 and 7; however, they did not examine the survival and index of each nodal station.

In the $\mathrm{AC}$ group, the subsequent lymph nodes after nos. $1,2,3,7$ and the lower mediastinal nodes were the lymph nodes along the proximal splenic artery (no. 11p). The previous studies in junctional AC demonstrated similar results, with the third to sixth priority being recorded among the abdominal lymph nodes [14-18]. In contrast, the metastatic incidence was only $2.9 \%(1 / 34)$ in the SCC group, and the calculated index was 0 in this study, suggesting that the benefit of dissecting no. $11 \mathrm{p}$ may differ depending on the histological type.

Prophylactic paraaortic node dissection (PAND) is not recommended for advanced gastric cancer based on the results of a phase III trial performed in Japan (JCOG9501) [21]; however, this result may not be applicable for patients with junctional adenocarcinoma, because junctional adenocarcinoma was not included in that trial. In the present study, the index of $16 \mathrm{a} 2$ was relatively high, with seventh 
priority in AC. These results suggest that $16 \mathrm{a} 2$ should be a target of nodal dissection in AC patients. On the other hand, the paraaortic nodes were not frequently dissected in the SCC group. Although the metastatic incidence seems to be similar in the AC and SCC groups, reliability for SCC was low. The index of the paraaortic nodes for the SCC group could not be calculated in this study. It remained unclear whether $16 \mathrm{a} 2$ should be a target of nodal dissection in SCC patients.

The present study is associated with several limitations that should be taken into consideration when interpreting the results. First, the number of patients with SCC was relatively small. The index of the nodes might therefore be unreliable, especially in the nodes that were dissected in a small number of patients, although we excluded the nodes that were not frequently dissected ( $<20$ cases) when calculating the index. However, the index of SCC was clearly separated for the nodes, with some values being high and others being 0 . The only lymph nodes showing some value of the index were nos. 1, 2, 3, 7, 9 and the lower mediastinal lymph nodes. Other nodes, such as nos. 8a, 11p and the middle to upper mediastinal nodes, all had values of 0 , which was caused by the very low metastatic incidence and no survivors in nos. $8 \mathrm{a}$ and $11 \mathrm{p}$, and due to the relatively high metastatic incidence but no survivors in the middle to upper mediastinal nodes. The order of lymph nodes with regard to the index might change by increasing the sample size, but the recommended nodes that should be dissected would not be changed. Second, some patients received neoadjuvant chemotherapy, which could affect the incidence of lymph node metastasis and the index. However, it has not been confirmed that chemotherapy selectively induces a pathological complete response of the nodes by examining the different locations of the lymph nodes. Although the order of the nodes might be changed by neoadjuvant chemotherapy, the possibility of such a change would be low.

The third issue is selection bias. This study was conducted mainly by the surgeons belonging to the upper gastrointestinal team whose specialty is the esophagus and the stomach. In some institutions, however, these two organs are treated separately and only gastric surgeons participated in this study. Although tumors whose center is located between $1 \mathrm{~cm}$ above and $2 \mathrm{~cm}$ below the junction are usually treated by gastric surgeons, the patients who were operated on by esophageal surgeons may not have been included at some institutions in this study. Fourth, in some cases it was very difficult to determine the EJ junction. In this study, in difficult cases physicians finally determined the location of the EJ junction by their own experience and subjective findings. However, we did not collect data on the degree of ambiguity in the measurements and cannot exclude such cases.
In conclusion, the histological type of junctional cancer was not a significant determinant for the major priority of nodal dissection. Nodal dissection achieved by proximal gastrectomy and lower esophagectomy enables dissection of the lymph node stations with the first to fifth highest indices and therefore should be a minimal requirement for the treatment of junctional cancer regardless of the histology of the primary tumor.

Conflict of interest Nothing to declare.

\section{References}

1. Blot WJ, Devesa SS, Kneller RW, Fraumeni JF Jr. Rising incidence of adenocarcinoma of the esophagus and gastric cardia. JAMA. 1991;265:1287-9.

2. Devesa SS, Blot WJ, Fraumeni JF Jr. Changing patterns in the incidence of esophageal and gastric carcinoma in the United States. Cancer. 1998;83:2049-53.

3. Lerut T, Decker G, Coosemans W, De Leyn P, Decaluwe H, Nafteux P, et al. Quality indicators of surgery for adenocarcinoma of the esophagus and gastroesophageal junction. Recent Results Cancer Res. 2010;182:127-42.

4. Corley DA, Buffler PA. Oesophageal and gastric cardia adenocarcinomas: analysis of regional variation using the Cancer Incidence in Five Continents database. Int $\mathrm{J}$ Epidemiol. 2001;30:1415-25.

5. Holmes RS, Vaughan TL. Epidemiology and pathogenesis of esophageal cancer. Semin Radiat Oncol. 2007;17:2-9.

6. Gholipour C, Shalchi RA, Abbasi M. A histopathological study of esophageal cancer on the western side of the Caspian littoral from 1994 to 2003. Dis Esophagus. 2008;21:322-7.

7. Morita M, Yoshida R, Ikeda K, Egashira A, Oki E, Sadanaga N, et al. Advances in esophageal cancer surgery in Japan: an analysis of 1000 consecutive patients treated at a single institute. Surgery. 2008;143:499-508.

8. Siewert JR, Stein HJ. Classification of adenocarcinoma of the oesophagogastric junction. Br J Surg. 1998;85:1457-9.

9. Stein HJ, Feith M, Siewert JR. Cancer of the esophagogastric junction. Surg Oncol. 2000;9:35-41.

10. Sobin LH, Compton CC. TNM seventh edition: what's new, what's changed: communication from the International Union Against Cancer and the American Joint Committee on Cancer. Cancer. 2010;116:5336-9.

11. Japanese Gastric Cancer Association. Japanese classification of gastric carcinoma: 3rd English edition. Gastric Cancer. 2011;14:101-12.

12. Association JGC. Japanese gastric cancer treatment guidelines 2010 (ver. 3). Gastric Cancer. 2011;14:113-23.

13. Sasako M, McCulloch P, Kinoshita T, Maruyama K. New method to evaluate the therapeutic value of lymph node dissection for gastric cancer. Br J Surg. 1995;82:346-51.

14. Yamashita H, Katai H, Morita S, Saka M, Taniguchi H, Fukagawa T. Optimal extent of lymph node dissection for Siewert type II esophagogastric junction carcinoma. Ann Surg. 2011;254:274-80.

15. Mine S, Sano T, Hiki N, Yamada K, Nunobe S, Yamaguchi T. Lymphadenectomy around the left renal vein in Siewert type II adenocarcinoma of the oesophagogastric junction. Br J Surg. 2013;100:261-6.

16. Fujitani K, Miyashiro I, Mikata S, Tamura S, Imamura H, Hara J, et al. Pattern of abdominal nodal spread and optimal abdominal lymphadenectomy for advanced Siewert type II adenocarcinoma 
of the cardia: results of a multicenter study. Gastric Cancer. 2013;16:301-8.

17. Goto H, Tokunaga M, Sugisawa N, Tanizawa Y, Bando E, Kawamura $\mathrm{T}$, et al. Value of splenectomy in patients with Siewert type II adenocarcinoma of the esophagogastric junction. Gastric Cancer. 2013;16:590-5.

18. Hasegawa S, Yoshikawa T, Rino Y, Oshima T, Aoyama T, Hayashi $\mathrm{T}$, et al. Priority of lymph node dissection for Siewert type II/III adenocarcinoma of the esophagogastric junction. Ann Surg Oncol. 2013;20:4252-9.

19. Yabusaki H, Nashimoto A, Matsuki A, Aizawa M. Comparison of the surgical treatment strategies for Siewert type II squamous cell carcinoma in the same area as esophagogastric junction carcinoma: data from a single Japanese high-volume cancer center. Surg Today. 2014;44:1522-8.

20. Kakeji Y, Yamamoto M, Ito S, Sugiyama M, Egashira A, Saeki $\mathrm{H}$, et al. Lymph node metastasis from cancer of the esophagogastric junction, and determination of the appropriate nodal dissection. Surg Today. 2012;42:351-8.

21. Sasako M, Sano T, Yamamoto S, Kurokawa Y, Nashimoto A, Kurita A, et al. D2 lymphadenectomy alone or with para-aortic nodal dissection for gastric cancer. $N$ Engl $J$ Med. 2008;359:453-62. 